\title{
Metamaterials subwavelength terahertz resonant cavities
}

\author{
M. Al-Rubaiee ${ }^{1,2^{*}}$ (D, A. H. Al-Janabi ${ }^{3}$, S. C. Fleming ${ }^{1}$ and A. Argyros ${ }^{1}$
}

\begin{abstract}
One of the unique properties of metamaterials is the ability to manipulate electromagnetic waves at subwavelength scales, made possible by their structure on these scales. Here, rather than consider effective bulk properties, we consider the properties of microscopic features based on considering resonant unit cells. We used wire array metamaterials to form localized resonant cavities by changing the resonance frequency of one or more unit cells, surrounded by unchanged unit cells that do not support resonance for the propagating mode (i.e. forming a band gap). We validate our approach experimentally with electromagnetic waves in the terahertz range, demonstrating and characterizing subwavelength resonant cavities in this range. These resonant cavities can pave the way for ultra-compact subwavelength waveguides and other optical components.
\end{abstract}

Keywords: Metamaterials, Subwavelength cavities, Resonator

\section{Introduction}

Wave propagation control is of fundamental interest in many areas of engineering and physics. One of the designs that has proven its efficiency in controlling electromagnetic waves is the photonic crystal $[1,2]$. This artificial media requires structures with dimensions comparable to the wavelength (relatively large dimensions) [3-5]. Metamaterials can control wave propagation at deep subwavelength spatial scales potentially enabling applications in nano-sensors directly on chip [6-8]. They are usually metal-dielectric composites capable of achieving unique electromagnetic properties not encountered in natural materials [9-11]. This deep subwavelength feature of metamaterials gives an advantage over photonic crystals in terms of wave manipulation [3, 12, 13]. Metamaterials have typically been studied for their effective bulk properties, such as negative refraction [14-16]. However, for some properties it is necessary to go beyond this effective medium paradigm; in this work a microscopic approach has been used to

\footnotetext{
* Correspondence: mohanad.alrubaiee@kus.edu.iq

'Institute of Photonics and Optical Science (IPOS), School of Physics, The University of Sydney, Sydney, NSW 2006, Australia

${ }^{2}$ Al-Karkh University of Science, Baghdad 10003, Iraq

Full list of author information is available at the end of the article
}

study metamaterials based on resonant unit cells [12, 14]. In photonic crystals, light can propagate in linear defects, typically comprised of removed unit cells [15, 16]. In wire array metamaterials, waveguiding and finite sub-wavelength resonators can be achieved by changing the resonance frequency of one or more unit cells, surrounded by unchanged unit cells that do not support the resonance for the propagating mode, thus forming a band gap (when measuring transmission through the sample, there is a range of frequencies with low transmission which we refer to as bandgap, while the range of frequencies with high transmission we refer to as resonance) [17].

Here, we consider the case of defects in finite-size wire array metamaterials. The metamaterial consists of twodimensional periodic arrays of wire segments, with each wire perpendicular to the plane of the array, the wire diameter $d$ and spacing $a$ being much smaller than the wavelength. The length of the wire segments $L_{0}$ however is not sub-wavelength: each wire segment supports resonances at frequencies for which an integer number of half-wavelengths corresponds to the wire's length - the Fabry-Perot resonances [18]. Changing the resonance frequency can thus be achieved simply by changing the

Springer Open

(c) The Author(s). 2021 Open Access This article is licensed under a Creative Commons Attribution 4.0 International License, which permits use, sharing, adaptation, distribution and reproduction in any medium or format, as long as you give appropriate credit to the original author(s) and the source, provide a link to the Creative Commons licence, and indicate if changes were made. The images or other third party material in this article are included in the article's Creative Commons licence, unless indicated otherwise in a credit line to the material. If material is not included in the article's Creative Commons licence and your intended use is not permitted by statutory regulation or exceeds the permitted use, you will need to obtain permission directly from the copyright holder. To view a copy of this licence, visit http://creativecommons.org/licenses/by/4.0/. 
length of the wires, according to the following equation [12]:

$$
f_{m}=\frac{m c}{2 n L_{0}}
$$

where $c$ is the speed of light, $m$ is an integer larger than or equal to $1, n$ is the background refractive index.

In this periodic array, a defect is introduced by having one or several segments of different length ' with resonant frequencies $f_{m}^{\prime}$. At the resonant frequencies of these wires, the fields can be strongly confined at the defect site.

The other parameters, such as diameter and spacing, do not play a major part in the resonance, although these parameters give some control over the tradeoff between quality factor and mode volume. By reducing the diameter and spacing, the quality factor and mode volume of the defect mode will both increase. The defect mode's field is typically confined to a small area around the wire (S) given by eq. (2), where the wire diameter is limited to be larger than a few skin depths [14]:

$$
S=(2 a)^{2}
$$

\section{Methodology}

Subwavelength cavities using metal wire array metamaterials were prepared and characterized as follows. Subwavelength resonators and waveguides, operating in the microwave region, made of an array of copper wires of relatively large dimensions have been demonstrated in our previous work [19]. In the current work and in order to move to frequencies higher than microwave (i.e. $\mathrm{THz}$ or optical frequencies), the length of the wire array must be much smaller, according to eq. (1) as well the wire diameter (d) which should preferably be maintained within $\mathrm{a} / 4$ to $\mathrm{a} / 8$ [14]. Drawn metamaterial fibers seemed practical to be used for preparing a wire array on small dimensions; these drawn metamaterial fibers consist of metal wires embedded, typically, in polymer. Metamaterial fibers, consisting of indium wires in PMMA and/or Zeonex, have been drawn with different dimensions using the fiber drawing process.

An appropriate metamaterial fiber was chosen from samples fabricated for earlier work by fibre drawing [20]. It comprised a Zeonex host material incorporating multiple indium wires. The overall diameter was $950 \mu \mathrm{m}$, without cladding, the wire spacing was $50 \mu \mathrm{m}$, the wire diameter $22 \mu \mathrm{m}$, with total of around 450 wires. The choice of this metamaterial fiber was based on its dimensions and material. It is not too small for handling: as very short samples are required, samples with small diameter would be hard to handle, polish and etch.
Zeonex has less loss than PMMA in the wavelength region of interest.

Several samples with different lengths, ranging from 430 to $275 \mu \mathrm{m}$ were prepared by cutting short sections from this fibre, then polishing the sample faces with polishing papers with a sequence of roughnesses of $3 \mu \mathrm{m}$, $1 \mu \mathrm{m}$ and $0.3 \mu \mathrm{m}$. This is to make them as regular and smooth as possible and to finely control the sample length. A microscope image of the face of the metamaterial fiber, after sample preparation by polishing, is shown in Fig. 1.

To characterize the behavior of this metamaterial we need to measure the spectral transmission of the sample in the $\mathrm{THz}$ range to determine the resonance and bandgap frequencies. The transmission of the samples was measured using a $\mathrm{THz}$ far field detector time domain spectroscopy (TDS) system. This system comprised a Coherent MIRA Ti:Sapphire mode locked laser, operating at $800 \mathrm{~nm}$, producing $180 \mathrm{fs}$ pulses at $86 \mathrm{MHz}$ repetition rate with average power of approximately $600 \mathrm{~mW}$ exciting $\mathrm{THz}$ antennas for source and detection. Generally, the detection antenna was placed in the far field to determine overall spectral properties with good signal strength. To measure spatial distributions with good precision, near field detection was also used. The samples were attached to a metal disc with a single hole (the same size as the sample diameter) in the center to block unwanted signal during transmission measurement and allow only the signal that passed through the sample to reach the detector. The transmission of two samples ( $430 \mu \mathrm{m}$ and $375 \mu \mathrm{m}$ length) were measured using the $\mathrm{THz}$ far field system to identify the resonance and bandgap frequencies of each wire array metamaterial sample (these length values are not especially significant; these samples were chosen because of their uniformity in term of thinness and face polishing). The expected resonance for the two samples, calculated using eq. (1), where $n=$ 1.53 for Zeonex and $\mathrm{m}=2$, gives a resonance frequency, $\mathrm{f}$, for the first sample of $0.455 \mathrm{THz}$, and $0.522 \mathrm{THz}$ for the second sample. The measured transmission spectrum, along with resonance and bandgap frequency regions, are shown in Fig. 2, for these sample lengths at $\mathrm{m}=2$ and show a good match with the frequencies calculated above.

In order to demonstrate a subwavelength resonator using our samples, one or more wires need to be shorter than the other wires of the sample to shift their resonance frequencies to the bandgap side of the longer wires. Etching was chosen as a practical method to shorten one or more wires. We chose hydrochloric acid, as it is readily available and used for metal etching, and importantly it does not affect the polymer. To etch a sample to a certain depth, it is essential to know the etching rate. This was obtained by placing many samples (all with wire 


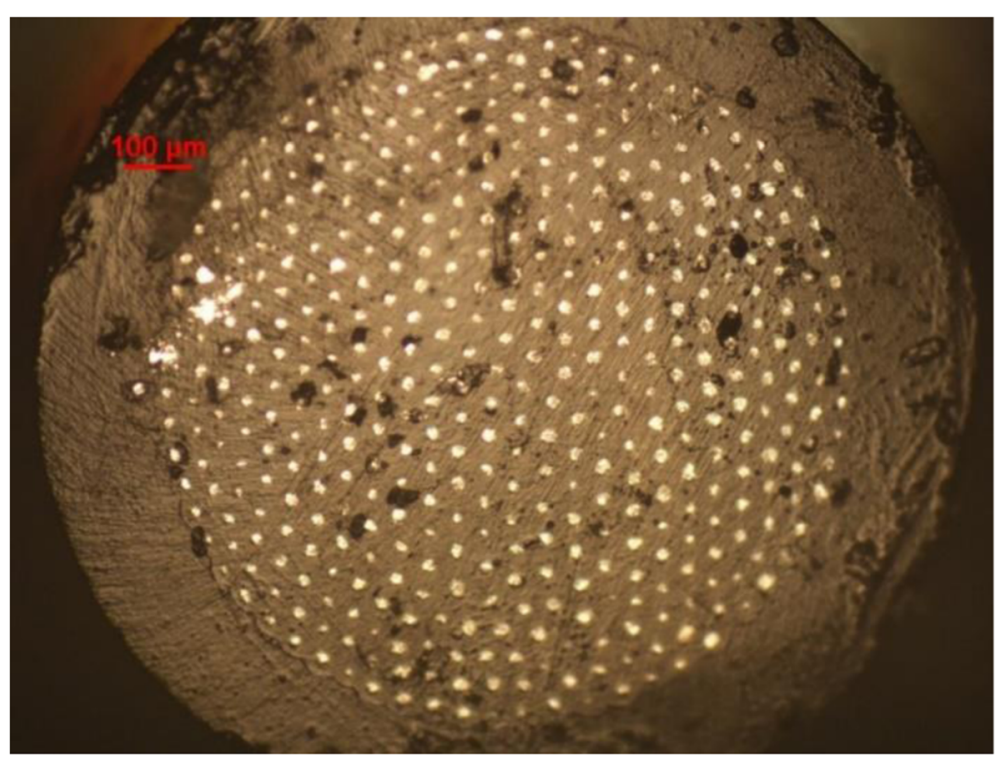

Fig. 1 Microscope image of prepared sample of metamaterial fibre after polishing, bright spots are indium (The overall diameter is $950 \mu m$, without cladding, wire spacing is $50 \mu \mathrm{m}$, wire diameter is $22 \mu \mathrm{m}$ )

diameters around $22 \mu \mathrm{m})$ for different times in hydrochloric acid then measuring the etching depth for each interval. As shown in Fig. 3, for each etching time there is a fairly wide etching depth range, as indicated by the vertical red bars. Nevertheless, there is a clear, approximately linear, trend after the first $30 \mathrm{~min}$. For the initial period there is no etching apparent, presumably due to the time required to etch through the oxidation layer. This oxidation layer appeared with all drawn metamaterial samples. The etching rate, after this initial period, is approximately $50 \mathrm{~nm} / \mathrm{s}$.

To make only a few wires shorter than the others, a mask is required. This was performed on the two faces of the sample using transparent plastic tape, which does not dissolve in hydrochloric acid, and a small hole was made in the tape on one of the faces to allow the hydrochloric acid to reach the indium wires. The smallest obtained hole was around 50-
$60 \mu \mathrm{m}$, this dimension covers an area of two adjacent wires. The target is for the wires to be shorter by $5 \%$ to $12 \%$ in order for their resonance to be in the bandgap of the sample.

Two samples were prepared for etching, with lengths $375 \mu \mathrm{m}$ and $275 \mu \mathrm{m}$. They were placed in hydrochloric acid for $35 \mathrm{~min}$ for a target etch depth of $20 \mu \mathrm{m}$. The etched samples were removed from the acid and the mask was removed, then the samples were cleaned with water to eliminate any remaining acid on the sample and inside the holes, and then dried. The $275 \mu \mathrm{m}$ sample was placed under the microscope; the expected two shortened wires are marked with the red line near the center of Fig. 4.

\section{Results}

The transmission of both the $375 \mu \mathrm{m}$ and $275 \mu \mathrm{m}$ samples after etching was measured in the $\mathrm{THz}$ time domain spectroscopy system, as shown in Fig. 5.
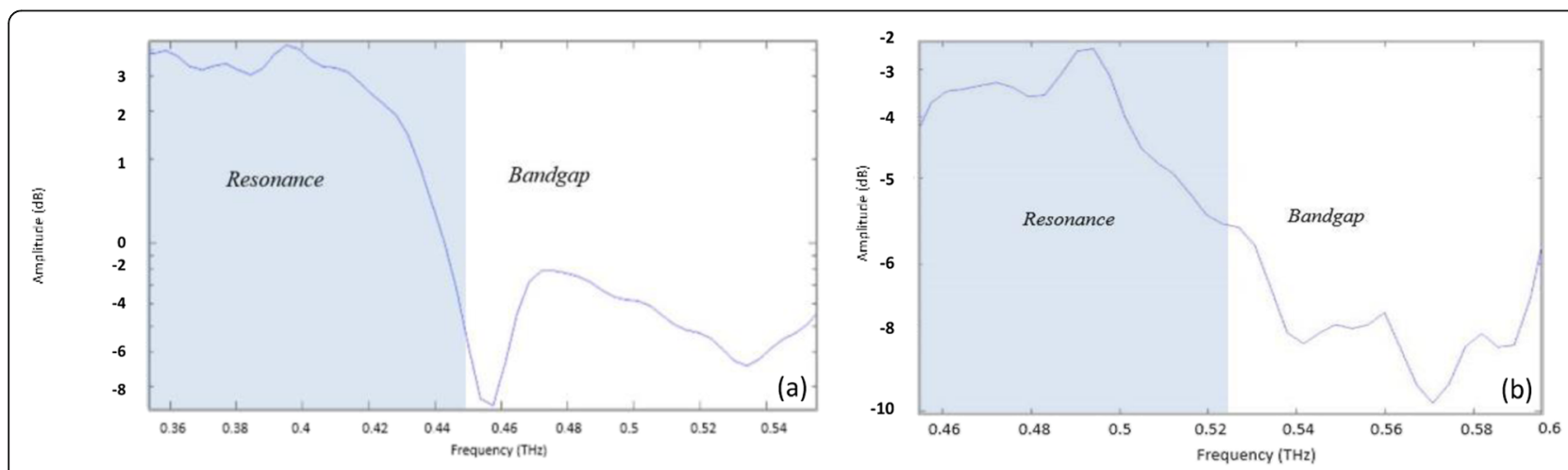

Fig. 2 a THz spectral transmission of (a) $430 \mu \mathrm{m}$ length sample, and (b) $375 \mu \mathrm{m}$ length sample 


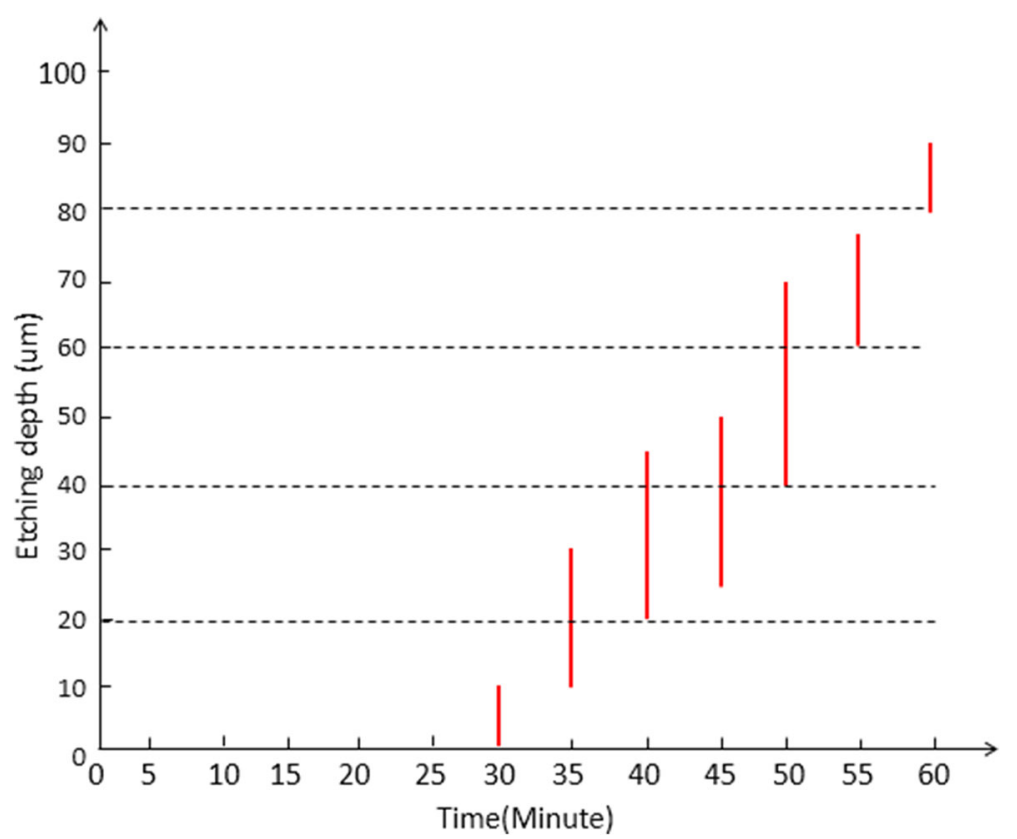

Fig. 3 Determination of etching depth range (vertical red bar) for indium wires using hydrochloric acid for different etching durations

For both samples a new, narrow, resonance peak appears in the bandgap, as shown in Fig. 5. The samples used in Figs. 5(a) and Fig. 2(b) are both $375 \mu \mathrm{m}$ long, permitting a direct comparison (the difference in transmission levels between the unetched regions and the etched regions is due to difference in experiment circumstances and laser power). It is clear that there is another new resonance peak located around $0.56 \mathrm{THz}$. Figure $5(\mathrm{~b})$ is for a shorter sample where the resonance frequencies of the sample are below $0.71 \mathrm{THz}$; however, there is another narrow resonance peak around 0.755 $\mathrm{THz}$. These resonance peaks $(0.56 \mathrm{THz}$ for the $375 \mu \mathrm{m}$ sample and $0.755 \mathrm{THz}$ for the $275 \mu \mathrm{m}$ sample) are the resonances of the shorter wires. From the resonance frequency eq. (1), the length of the shorter wires in the $375 \mu \mathrm{m}$ sample can be determined to be around $355 \mu \mathrm{m}$, which is $20 \mu \mathrm{m}$ shorter than the other wires of the sample and consistent with the range of the etching depths

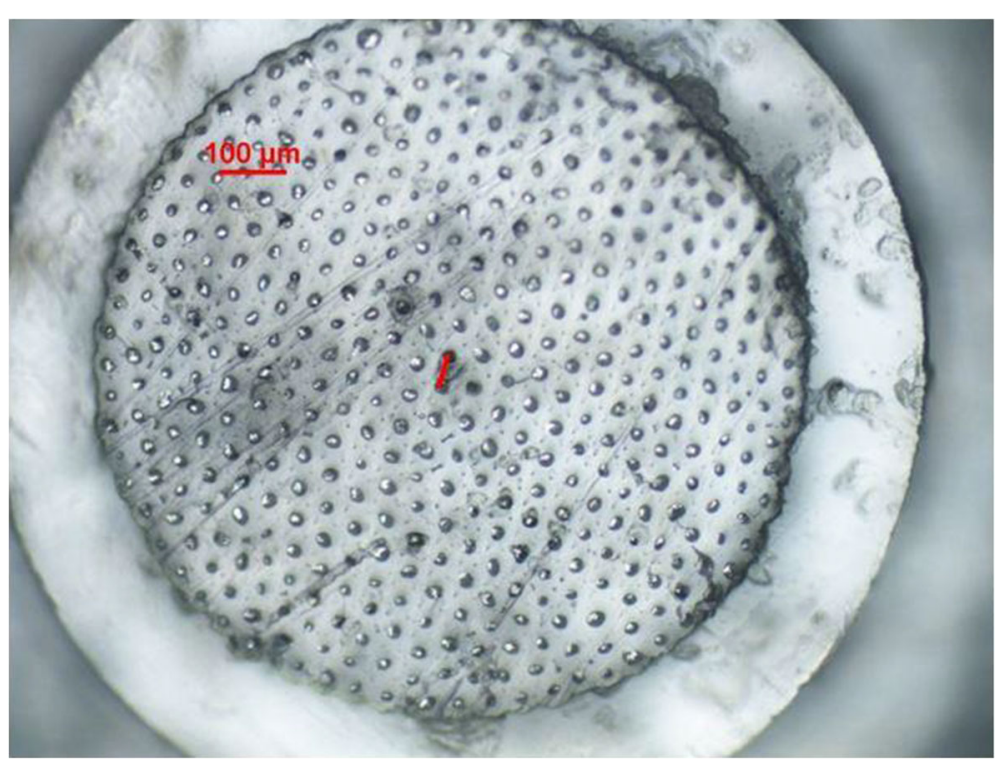

Fig. 4 Metamaterial resonator sample after etching two wires (indicated with red line) 

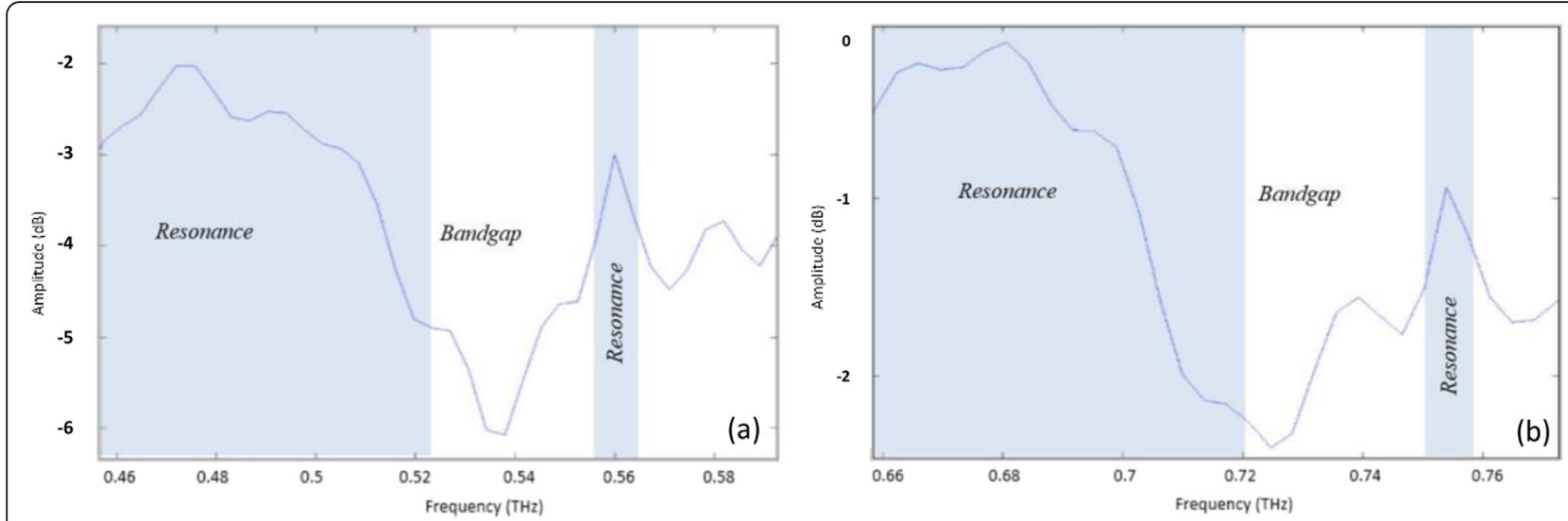

Fig. 5 Transmission of wire array metamaterial resonators with two etched wires for (a) $375 \mu \mathrm{m}$ length wires, and (b) $275 \mu \mathrm{m}$ length wires

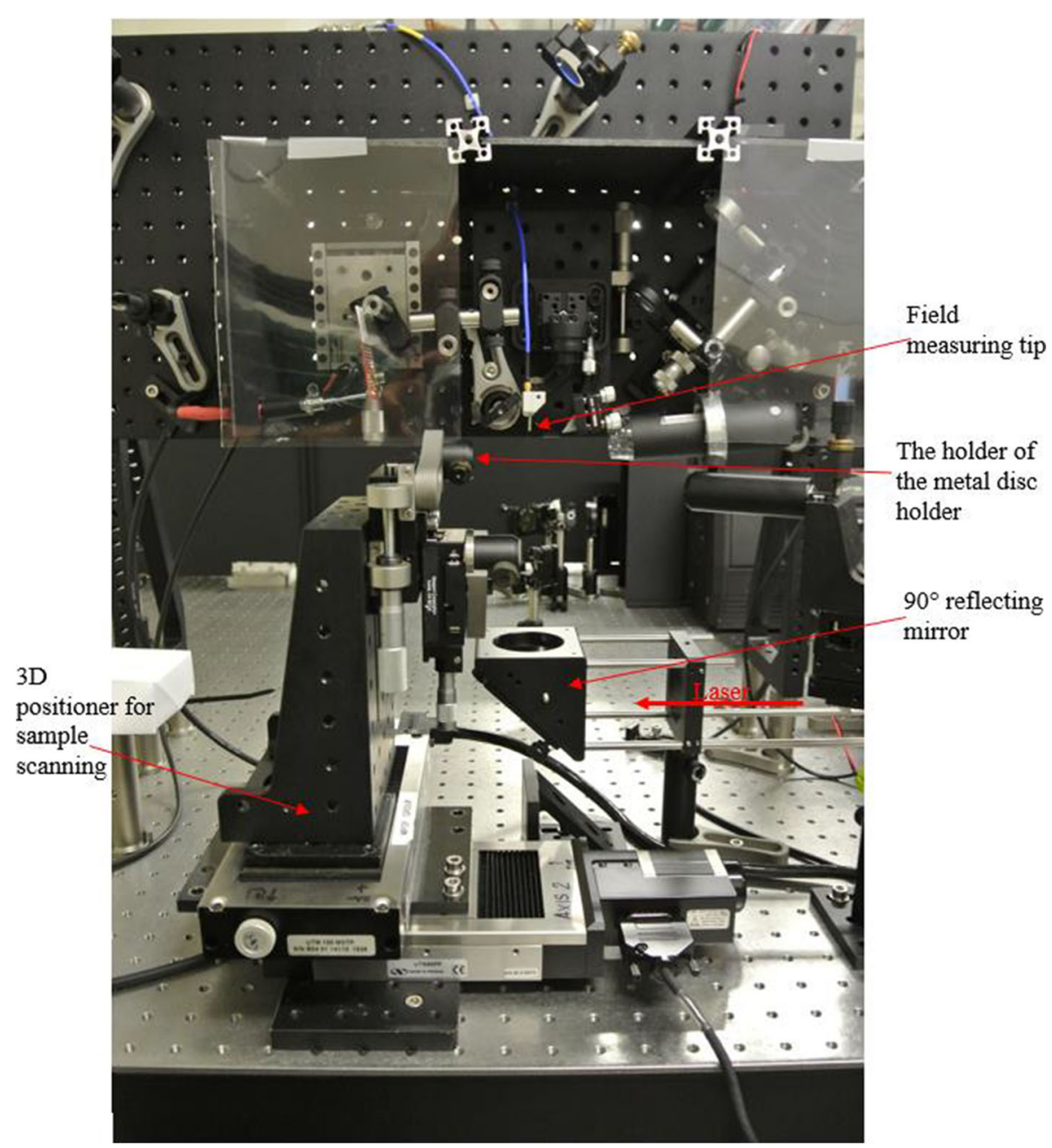

Fig. 6 Terahertz near field detector system 


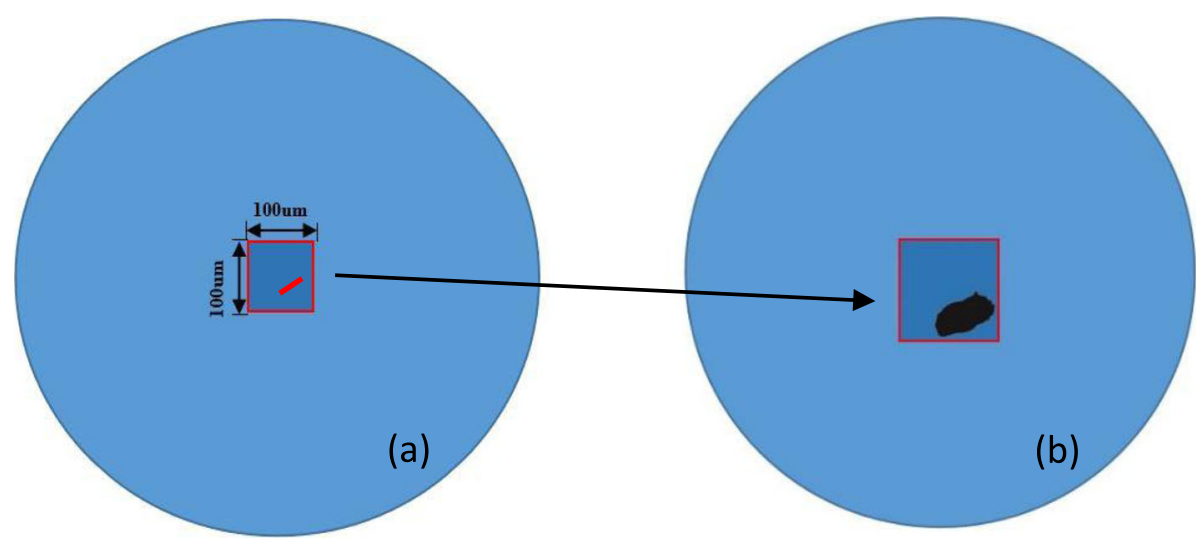

Fig. 7 Illustration of (a) the $100 \times 100 \mu \mathrm{m}$ area of the $275 \mu \mathrm{m}$ length sample scanned using the near field detector (short wires indicated in red). $\mathbf{b}$ the region (the dark area) of increased transmission at frequencies around $0.75 \mathrm{THz}$, in the vicinity of the short wires

for the 35 min interval $(10-30 \mu \mathrm{m})$. For the $275 \mu \mathrm{m}$ sample the estimated length from the resonance frequency peak is around $260 \mu \mathrm{m}$, which is $15 \mu \mathrm{m}$ shorter than the other wires of the sample and again consistent with the etching depth range.

Far field detection was used to determine the transmission of the whole sample (as one part). To map the field near the defects, we use a raster-scanning near-field photoconductive $\mathrm{THz}$ antenna (TeraSpike TD-800-Z-HR), excited by the same Ti:Sapphire laser, also using time-domain spectroscopy. The near field antenna measures the longitudinal field parallel to the wires, with a resolution up to $3 \mu \mathrm{m}$, but resolution is in practice limited by the distance from the sample and the decay of evanescent waves. This near field detector system (shown in Fig. 6) was used for measuring the transmission of the $275 \mu \mathrm{m}$ wire array metamaterial sample.

The near field was scanned over an area of 100 by $100 \mu \mathrm{m}$ in the center of the sample (the square area with red borders shown in Fig. 7(a)) containing the area where the two shorter wires are located. This area was divided into 36 measurement points (a 6 by 6 array), the step between adjacent points was $20 \mu \mathrm{m}$. When the transmission was measured there was a noticeable increase in the transmission between 0.74 $\mathrm{THz}$ and $0.76 \mathrm{THz}$ in the region where the shortened wires were located (the dark area (Fig. 7(b)).

The transmission spectra at all scanned points of the unmodified area were similar (see the red curve in Fig. 8). However, the transmission spectra at all points in the dark area (in Fig. 7b), where the two shorter wires are

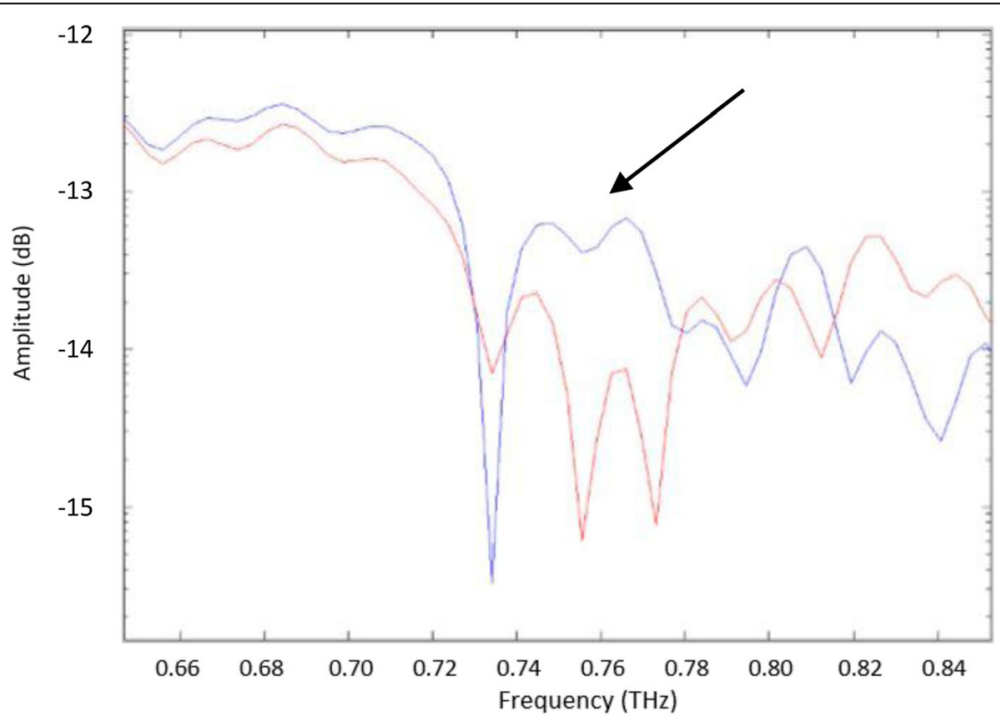

Fig. 8 Transmission spectra at points across the metamaterial for unmodified wire region (red curve) and vicinity of the etched wire region (blue curve). The Black arrow indicates the resonance that appear after etching 


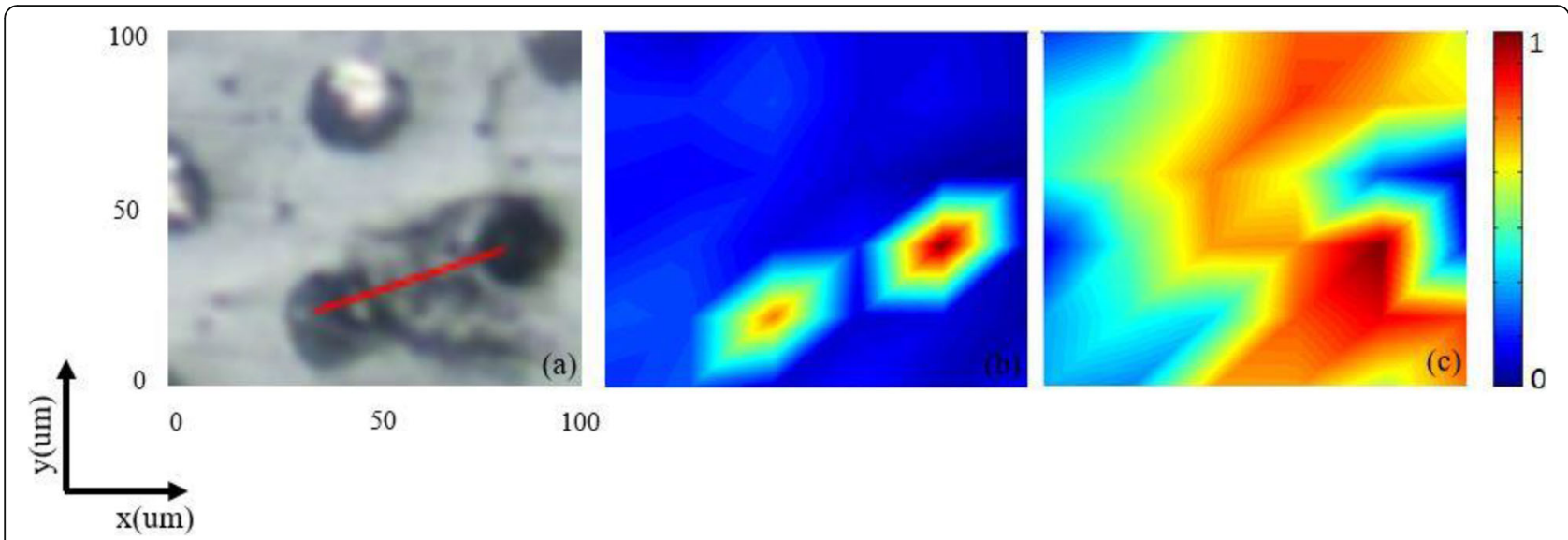

Fig. 9 a The $100 \times 100 \mu \mathrm{m}$ scanned area where the two shortened wires are located, (b) the field intensity (linear scale) at $0.755 \mathrm{THz}$, and (c) at $0.725 \mathrm{THz}$

located, are different (see the blue curve in Fig. 8). In this region two combined resonance peaks can be seen, the first around $0.75 \mathrm{THz}$ and the second around $0.77 \mathrm{THz}$

The near field detection technique gives the ability to make spatial measurements as it scans point by point (this different measurement setup and technique is the reason for the substantial amplitude difference between Fig. 8 and Fig. 5). Two resonance peaks of the two shortened wires can be seen. This is because the etching process is not very accurate (as can be seen in Fig. 3, an etching time of $35 \mathrm{~min}$ can give etching depths ranging from 10 to $30 \mu \mathrm{m}$ ). This is expected to be the reason behind the appearance of two resonance peaks as the two shorter wires are not exactly the same length so their resonance frequencies are slightly different.

An optical micrograph of the $100 \times 100 \mu \mathrm{m}$ scanned area is shown in Fig. 9(a), revealing the location of the two shortened wires. The field intensity plots for 0.755
$\mathrm{THz}$ and $0.725 \mathrm{THz}$ are shown in Fig. 9 (b) and (c) respectively. The first frequency is located in the bandgap region of the longer wires and in the resonance region for the shorter wires. Thus, it will resonate only at the frequency associated with the two short wires and transmission will be forbidden for the other wires; this expected behavior was observed, as shown in Fig. 9(b). The second frequency is located in the resonance region of the sample so propagation across the measurement area was observed, as shown in Fig. 9(c).

As can be seen from Fig. 9(b) the shorter wires act as subwavelength cavities for specific frequencies, otherwise rejected by the whole sample. By etching a subset of wires, we demonstrated confinement in a volume of $\lambda^{3} /$ 400 of the free space wavelength. These subwavelength cavities can be used for subwavelength waveguiding where the shorter wires can be arranged in many shapes such as line, V (as shown in Fig. 10) or other complex shapes. By exciting one side of the metamaterial with a
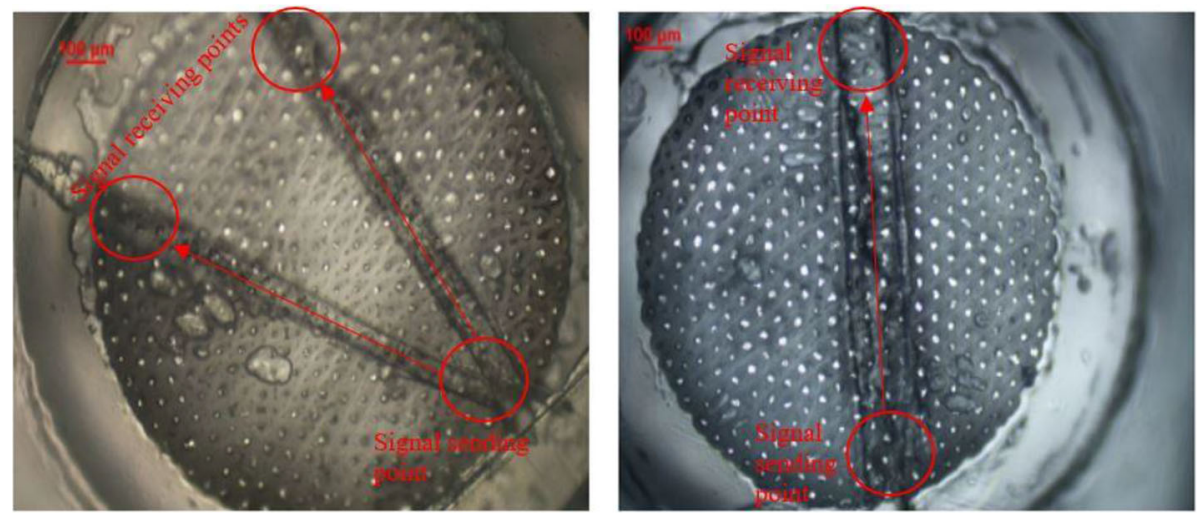

Fig. 10 a proposed $V$ shape subwavelength waveguide, (b) Proposed line shape subwavelength waveguide 
specific frequency located in the bandgap of the longer wires and in the resonance region of the shorter wires, efficient waveguiding can be obtained in these shapes. (The wider channel has more wires resulting in a higher probability of coupling with adjacent wires, since each wire will have two adjacent wires instead of one). However, the process for making many wires shorter using etching was difficult and had poor reproducibility. The etching process, as can be seen in Fig. 3, gives a large range of etching depths for each specific duration, which results in different lengths for the etched wires. This is a problem that will need to be addressed in future work because mismatched lengths may greatly reduce coupling efficiency between the wires.

\section{Conclusion}

We experimentally demonstrated the resonance of subwavelength cavities in wire-array metamaterials for $\mathrm{THz}$ frequencies. We consider their properties from the perspective of the resonant unit cell rather than their periodic structure. Near field images show field confinement at the etched wires only (cavity resonance at $0.75 \mathrm{THz}(\lambda=400 \mu \mathrm{m})$ ), and in a volume $\lambda^{3} /$ 400 of the free space wavelength. These cavities show promise for subwavelength waveguiding and may pave the way for the design of ultra-compact components owing to their subwavelength scale. These materials can be tunable to operate at different frequencies by changing their length. However, further experimental development is needed to increase the coupling efficiency between neighboring wires which requires precise length matching between the wires. These wire array fibres have potential for applications in subwavelength waveguides and biological imaging using $\mathrm{THz}$ radiation.

\section{Acknowledgments}

We thank Dr. Jessienta Anthony and Dr. Richard Lwin for their technical support. We also thank both A/Prof. Boris Kuhlmey for his valuable critiques and advice during the work and Dr. Alessandro Tuniz whose metamaterial fiber we used for the samples we made. We acknowledge the Research \& Prototype Foundry Core Research Facility at the University of Sydney where the metamaterial was fabricated using facilities that are part of the NSW Node of the Australian National Fabrication Facility.

\section{Authors' contributions}

All authors contributed to the study conception and design. Material preparation, data collection and analysis were performed by Mohanad AlRubaiee, Abdulhadi Al-Janabi, Simon Fleming and Alexander Argyros. The first draft of the manuscript was written by Mohanad Al-Rubaiee and all authors commented on previous versions of the manuscript. All authors read and approved the final manuscript.

\section{Funding}

Not applicable.

\section{Availability of data and materials}

The datasets generated during and/or analysed during the current study are available from the corresponding author on reasonable request.

\section{Declarations}

\section{Competing interests}

Not applicable.

\section{Author details}

${ }^{1}$ Institute of Photonics and Optical Science (IPOS), School of Physics, The University of Sydney, Sydney, NSW 2006, Australia. ${ }^{2}$ Al-Karkh University of Science, Baghdad 10003, Iraq. Institute of Laser for Postgraduate Studies, University of Baghdad, Baghdad, Iraq.

Received: 31 January 2021 Accepted: 7 June 2021

Published online: 16 June 2021

\section{References}

1. L. O'Faolain, X. Yuan, D. Mclntyre, S. Thoms, H. Chong, R.M. De La Rue and T. F. Krauss, "Low-loss propagation in photonic crystal waveguides", Electron Lett, Vol. 42, No. 25, 1454-1455 (2006)

2. J.-B. Beguin, Z. Qina, X. Luana, and H. J. Kimblea, "Coupling of light and mechanics in a photonic crystal waveguide" Appl Phys Sci, , Vol. 47, 9 Novemebr (2020)

3. Zhao, J., Li, X., Zhong, L., Chen, G.: Calculation of photonic band-gap of one dimensional photonic crystal. J. Phys. Conf. Ser. 183, 012018 (2009). https:// doi.org/10.1088/1742-6596/183/1/012018

4. Beggs, D.M., O'Faolain, L., Krauss, T.F.: Accurate determination of the functional hole size in photonic crystal slabs using optical methods. Photonics Nanostruct. Fundam. Appl. 6(3-4), 213-218 (2008). https://doi. org/10.1016/j.photonics.2008.09.003

5. Xiaochuan Xu, Zeyu Panb, Chi-Jui Chunga, Ching-Wen Changa, Hai Yana, and Ray T. Chen, "Dielectric metamaterial waveguide for optical interconnect and sensing", SPIE, Vol. 1092, March (2019)

6. Xu, K., Chen, Y., Okhai, T.A., Snyman, L.W.: Micro optical sensors based on avalanching silicon light-emitting devices monolithically integrated on chips. Optic Mater Expr. 9, (2019) No. 10

7. Tapashree Roy, Edward T. Rogers, and Nikolay I. Zheludev, "Sub-wavelength focusing meta-lens", Opt Express, Vol. 21, No. 6, March (2013), 7577

8. P. Cheben, R. Halir, J.H. Schmid, J Čtyroký, D. Benedikovic, C. Alonso-Ramos, A. Ortega-Moñux, A. Sánchez-Postigo, D. González-Andrade, J. G. Wangüemert-Pérez, I. Molina-Fernández, A. V. Velasco, A. Herrero-Bermello, J. M. Luque-González, D. PereiraMartín, J. Lapointe, S. Janz, D.-X. Xu, D. Melat ,Y. Grinberg, S. Wang, M. Vachon, V. Vakarin, L. Vivien, J. Litvík, J. Müllerová and M. Dado, "Subwavelength Silicon Photonic Metamaterial Waveguide Devices", 2018 European conference on optical communication (ECOC), Rome, (2018), pp. 1-3

9. Osama T. Naman, Matthew R. New-Tolley, Richard Lwin, Alessandro Tuniz, A Hadi Al-Janabi, Inna Karatchevtseva, Simon C. Fleming, Boris T. Kuhlmey, and Alexander Argyros, "Indefinite media based on wire array metamaterials for the THz and mid-IR", Advanced Optical Materials, Vol. 1, No. 12. December (2013), 971-977

10. Basharin, A.A., Mavidis, C., Kafesaki, M., Economou, E.N., Soukoulis, C.M. Epsilon near zero based phenomena in metamaterials. Phys. Rev. 87, 155130 (2013)

11. Syed S. Bukhari, J Vardaxoglou and William Whittow, "A metasurfaces review: definitions and applications", Appl. Sci., Vol. 9, July (2019), 2727

12. Fabrice Lemoult, Nadège Kaina, Mathias Fink and Geoffroy Lerosey, "Wave propagation control at the deep subwavelength scale in metamaterials", Nat. Phys., Vol. 9, January (2012)

13. Fatma A. Sayed, Hussein A. Elsayed \& Arafa H. Aly, "Optical properties of photonic crystals based on graphene nanocomposite within visible and IR wavelengths" Opt. Quantum Electron., Vol. 52, October (2020)

14. Kaina, N., Lemoult, F., Fink, M., Lerosey, G.: Ultra small mode volume defect cavities in spatially ordered and disordered metamaterials. Appl. Phys. Lett. 102, 144104 (2013)

15. Adam Mock and Ling Lu, "Recent Optical and Photonic Technologies" January (2010), INTECH, Croatia, pp. 450

16. Joannopoulos, J.D., Villeneuve, P.R., Fan, S.: Photonic crystals: putting a new twist on light. Nature. 386(6621), 143-149 (1997). https://doi.org/10.1038/3 $86143 a 0$

17. Alexander Argyros, Jessienta Anthony, Xiaoli Tang, Mohanad Jamal, A Hadi Al-Janabi, Simon C Fleming, Boris T Kuhlmey, "Subwavelength Imaging and 
Cavities Using Wire Array Fibres" OSA (Asia Communications and Photonics), November (2015), ASu1C. 2

18. Rakhmanov, M., Savage Jr., R.L., Reitze, D.H., Tanner, D.B.: Dynamic resonance of light in Fabry-Perot cavities. Phys. Lett. A. 305, 239-244 (2002)

19. M Al-Rubaiee, A Alchalaby, H Al-Janabi, "Subwavelength wire array metamaterial microwave cavities", SPIE (Nanophotonics Australasia 2017), Vol. 10456, January (2018)

20. Alessandro Tuniz, Richard Lwin, Alexander Argyros, Simon C. Fleming, Boris T. Kuhlmey, "Fabricating Metamaterials Using the Fiber Drawing Method", J Vis Exp, Vol. 68, October (2012)

\section{Publisher's Note}

Springer Nature remains neutral with regard to jurisdictional claims in published maps and institutional affiliations.

\section{Submit your manuscript to a SpringerOpen ${ }^{\odot}$ journal and benefit from:}

- Convenient online submission

- Rigorous peer review

- Open access: articles freely available online

High visibility within the field

- Retaining the copyright to your article

Submit your next manuscript at $\boldsymbol{\nabla}$ springeropen.com 